\title{
Structural colors with angle-insensitive optical properties generated by Morpho-inspired 2PP structures
}

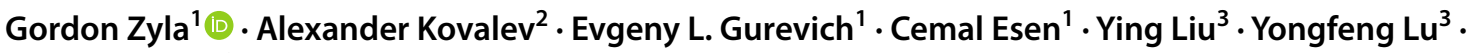 \\ Stanislav Gorb ${ }^{2} \cdot$ Andreas Ostendorf $^{1}$
}

Received: 8 June 2020 / Accepted: 19 August 2020

(C) The Author(s) 2020

\begin{abstract}
This paper describes how two-photon polymerization was used to generate biomimetic nanostructures with angle-insensitive coloration inspired by the blue butterflies of Morpho. Less angle dependence was achieved by engineering the structures with a certain degree of disorder, which delimited them from classical photonic crystals. Variations in the processing parameters enabled the color hue to be controlled. In this context, blue, green, yellow, and brown structures were demonstrated. Reflection spectra of the structures were simulated and studied experimentally in a broad range of incident angles. Additionally, a molding technique was performed as a potential scale-up strategy. The application of such biomimetic structures is discussed.
\end{abstract}

Keywords Biomimetic $\cdot$ Two-photon polymerization $\cdot$ Structural color $\cdot$ Photonic $\cdot$ Morpho butterfly

\section{Introduction}

Structural colors in nature result from interference, diffraction, or scattering of light. These colors can often be observed in animals and plants using them, for example, for camouflage [1], warning signals [2], or intraspecific sexual communication [3] to ensure the survival of the species. Therefore, the organisms use sophisticated micro- or nanometer surface structures on their epidermis [4-6]. The morphology of such biological surfaces is highly complex, and depending on the species, they can strongly differ. A good overview of the broad diversity of biological photonic surface structures can be found in the literature [7, 8]. Furthermore, the photonic systems of many species often exhibit disorder characteristics [5, 9], which can be attributed to single structural impurities or a complex spatial distribution of the structures and their periodicity on the organisms' surfaces. In this context, the disorder characteristics

Gordon Zyla

zyla@lat.rub.de

1 Applied Laser Technologies, Ruhr-Universität Bochum, 44801 Bochum, Germany

2 Functional Morphology and Biomechanics, Christian-Albrec hts-Universität Zu Kiel, 24098 Kiel, Germany

3 Department of Electrical and Computer Engineering, University of Nebraska-Lincoln, Lincoln, NE 68588, USA can cause extraordinary optical effects, e.g., the ultrawhite color of the Cyphochilus [10], the ultrablack color of the Bitis gabonica [11, 12], and the iridescent green color of the Chrysina gloriosa [13], because not only one but multiple physical mechanisms participate in the color formation. The most famous organisms producing structural coloration with unique optical properties though are probably the blue butterflies from the genus Morpho. Their intense coloration is primarily to appear highly attractive since its color appears less angle dependent to an observer than in conventional optical diffraction gratings [5, 14-18].

Ordered periodic nanostructures with a certain degree of the disorder can be produced by self-organization processes, such as self-assembly of colloidal particles [19] or laserinduced periodic surface structures (LIPSS) [20]. However, more flexible methods are required to mimic the complex surface structures of Morpho. For example, e-beam lithography [21-24], laser interference lithography [22], and multilayer deposition $[25,26]$ have been applied most recently. However, these methods are limited concerning the structure geometry produced since a vacuum atmosphere, or specific exposure masks are required for structuring. Therefore, the modification of structures is often associated with much effort. Consequently, true threedimensional (3D) structuring with ultrahigh spatial resolution might be beneficial in this case. Two-photon polymerization (2PP) provides this capability. Thus, structural colors inspired by nature could 
already be produced [27-30]. Arbitrary high resolution, 3D microstructures can be generated in a photosensitive material without specific exposure masks and vacuum [31, 32]. Therefore, compared to the techniques mentioned above, 2PP can be highly attractive not only for engineering but also for biology, as structural geometries can be easily modified to produce different photonic blueprints to analyze their effects on the optical properties and the color hue.

Two-photon polymerization was used to generate biomimetic surface structures inspired by the blue Morpho butterflies, including specific disorder characteristics. The artificial structures provided the same blue coloration and the same optical properties as the organisms. To replicate the nanoscaled features of these Morpho butterflies, we apply a specific polymerization method since the resolution of a conventional 2PP structuring process is too low. Furthermore, different colors were created by modifying the biomimetic surface structures using different average laser powers for 2PP. The morphology of all of these structures and their resulting optical properties were analyzed using scanning electron microscopy (SEM), light microscopy, and angle-resolved spectroscopy. Analytical calculations support the experimental results. Moreover, a potential application for anticounterfeiting and a method for scaling up using a soft-molding technique, are presented herein.

\section{Results}

\subsection{Surface structures of the blue Morpho butterflies and their biomimicry using 2PP}

The metallic blue color of many butterflies of the genus Morpho appears on the upper side of their four wings. The origin of the wing coloration in this genus is due to the cover scales on the wings, on which specific structures, referred to as ridges, can be identified (see Fig. 1a). The ridges also have lamellar substructures so that the surface morphology of a single wing scale is characterized by sophisticated, hierarchical micro- and nanostructures of chitin $(n=1.56)$ [14].

The primary physical mechanism for the formation of the blue color is based on multilayer interference. However,

\section{A Surface structures of $M$. didius:}

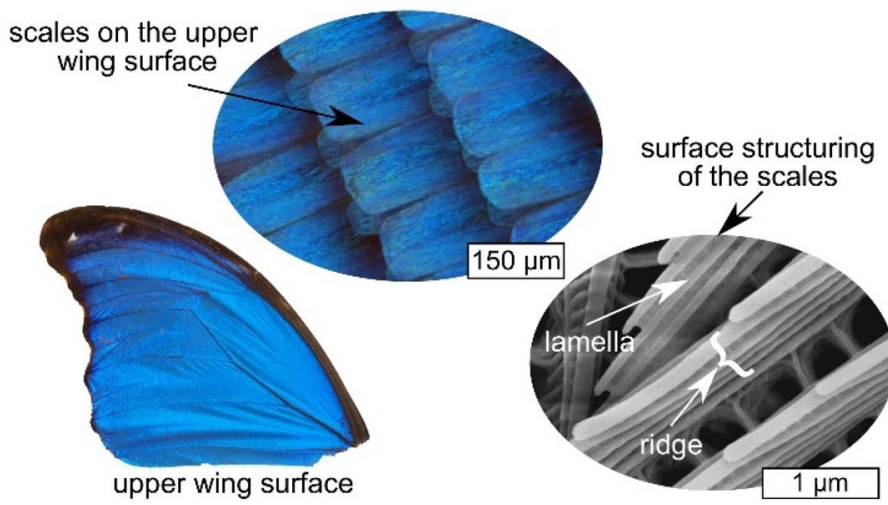

B Strategy for biomimicry by 2PP:

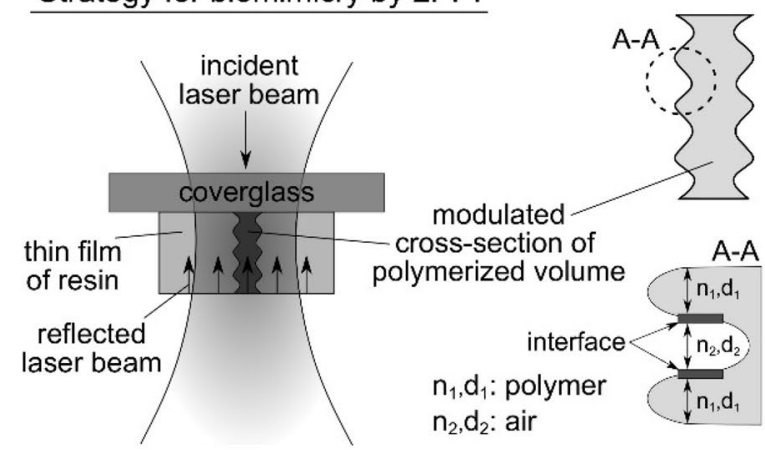

Fig. 1. Surface structures of $M$. didius and method for its biomimicry with 2 PP. a Blue structural coloration arises on the upper wing structure of $M$. didius due to multilayer systems with disorder characteristics. So-called ridges form the multilayer system with lamellar substructures on the structured scale surfaces. b The interference assisted polymerization method for $2 \mathrm{PP}$ is performed in a thin-film of

\section{Artificial structural color:}

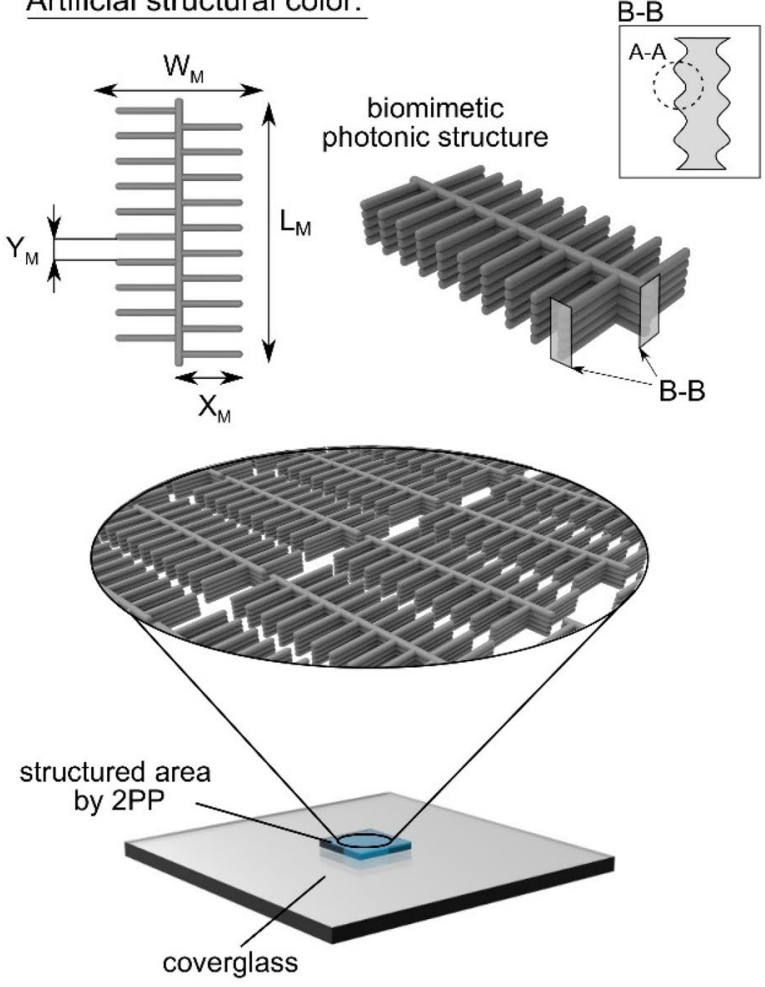

a photosensitive material. Here, substructures inside the cross-section of a polymer line arise from interference. These substructures form a multilayer system consisting of polymer and air, comparable to the lamellar Morpho ridges. c Illustration of the biomimetic photonic structure with modified cross-section. The biomimetic structures are aligned with each other to create a structural color for a specific area 
the color appears to be angle-insensitive to an observer in an angle range of $\pm 15^{\circ}$ [15], which in turn, does not match the optical properties of a conventional multilayer system. Here, the unique optical properties of these Morpho butterflies' wings are mostly due to disorder characteristics in the biological photonic system. Often, for instance, the ridges are of different heights of up to $400 \mathrm{~nm}[14,16,33,34]$, promoting the reflection of blue coloration [16]. In addition, the distance of individual lamellas are often not uniform because many of the ridges are tilted and distorted (see Fig. 1a). Therefore, inhomogeneity can be identified inside the multilayer systems, which significantly influences the angle-insensitivity of the observed coloration [16, 34, 35]. Moreover, the wing coloration of the blue Morpho butterflies is supported by so-called ground scales. These scales consist of melanin and absorb the transmitted light [36].

Mimicking the sophisticated photonic system of the blue Morpho butterflies with 2PP requires the application of a specific polymerization method. Otherwise, the resolution of the conventional 2PP process is too low [37]. In this context, the interference-assisted polymerization method, according to Mills et al. [38] and Liu et al. [39], proves to be particularly suitable, where $2 \mathrm{PP}$ is performed in a photosensitive thin film. Hence, a part of the incident laser radiation is reflected at the outer interface of the photosensitive material, which can interfere with the incident laser beam provided that the laser's coherence length is not exceeded [40]. As a result, a standing wave-like electromagnetic field is generated, modulating the cross-sectional geometry of a polymerized volume depending on the average laser power and the wavelength of the laser radiation [39]. Thus, polymer lines produced in this way can be used to build a multilayer system of polymer and air similar to the lamellar ridges of the blue Morpho butterflies (see Fig. 1b).

\subsection{Fabrication of artificial structural colors}

Experiments were performed with the conventional 2PP system, Photonic Professional GT2 (Nanoscribe GmbH, Karlsruhe, Germany). The laser emits ultrashort pulses approximately $100 \mathrm{fs}$ with a central wavelength of $780 \mathrm{~nm}$ and a repetition rate of $80 \mathrm{MHz}$. Its coherence length can be calculated to approximately $l_{\mathrm{c}}=30 \mu \mathrm{m}$ [40] assuming a spectral width of the emitted laser radiation of $20 \mathrm{~nm}$. Additionally, the device has a $63 \times$ microscope objective $(\mathrm{NA}=1.4$ with immersion oil, Zeiss, Oberkochen, Germany) to focus the laser radiation.

The interference-assisted 2PP method was performed layer by layer over the entire layer thickness of the photosensitive material. Each layer was polymerized with the use of an integrated galvo scanner deflecting the laser beam in the specific structural geometry produced. Afterwards, the high-precision axis system of Photonic Professional GT2 was used to adjust an axial offset between the individual layers, which was $200 \mathrm{~nm}$ continuously for all experiments. A mechanical axis system enables the manufacturing of multiple structures on a cover glass.

Thin-film layers of photosensitive material were previously prepared by spin coating using the organicinorganic hybrid polymer Femtobond 4B (Laser Zentrum Hannover e.V., Hannover, Germany, $n=1.51)$. Here, the aim was to create a thickness of $1 \mu \mathrm{m}$, which correlated with the average height of the blue Morpho butterflies' ridges [15]. To produce such a thin layer, the Femtobond $4 \mathrm{~B}$ had to be initially diluted with 2-propanol in a ratio of 1:3. Afterwards, the spin coating (SP150, SPSEurope, Putten, Netherlands) was conducted at a rotational speed of $1000 \mathrm{rpm}$ for $60 \mathrm{~s}$. The resulting film thickness was measured with a white light interferometer (TMS 1200, Polytec, Waldbronn, Germany). For this purpose, the thin films were previously polymerized by a super high-pressure mercury lamp (HB-10103AF, Nikon, Düsseldorf, Germany) for $24 \mathrm{~h}$.

To mimic the lamellar ridges of the blue Morpho butterflies and modify the biomimetic multilayer systems, we used different average laser powers to produce the structures presented in Fig. 1c. Single structures have a width of about $W_{\mathrm{M}}=4 \mu \mathrm{m}$, and a length of about $L_{\mathrm{M}}=11 \mu \mathrm{m}$. The parameters $X_{\mathrm{M}}$ and $Y_{\mathrm{M}}$ define the exposure paths for each layer. The distance between the horizontally illustrated lines is $Y_{\mathrm{M}}=1 \mu \mathrm{m}$. For the generation of a rectangular colored surface $\left(A=2 \mathrm{~mm}^{2}\right)$ with high color intensity, individual structures were arranged compactly with a lateral distance of $800 \mathrm{~nm}$. The manufacturing time was $12 \mathrm{~h}$ using a galvo scanner velocity of $1.4 \mathrm{~mm} / \mathrm{s}$. After finishing the polymerization process, the samples were rinsed for $30 \mathrm{~min}$ in 2-propanol.

The images presented in Fig. 2 clearly show the successful production of a blue, green, yellow and brown coloration. The color formation can be attributed to the multilayer system formed within the cross-sections of the individual lines (see SEM images in Fig. 2) since fully polymerized Femtobond 4B is generally transparent [30]. Furthermore, diffraction, as well-known from optical gratings, can be excluded as the origin of the colors, as proven by our previous work. In this work, we showed that varying the distance between individual polymer lines only affects the color intensity [30]. For color imaging, the structured areas were aligned planar on a sample holder below a light microscope (Eclipse LV 100 N, Nikon, Düsseldorf, Germany). Illumination was performed with an objective $(5 \times, \mathrm{NA}=0.15$, Nikon, Düsseldorf, Germany) at an angle of $0^{\circ}$, referring to the surface normal of the sample. Images were recorded at the same angle by an integrated camera (DS-Fi2, Nikon, Düsseldorf, Germany). A detailed overview of the average laser power values $\left(P_{\mathrm{a}}\right)$ used and the resulting average layer thicknesses of Femtobond 4B $\left(d_{1}\right)$ and air $\left(d_{2}\right)$ is given in Table 1. The 

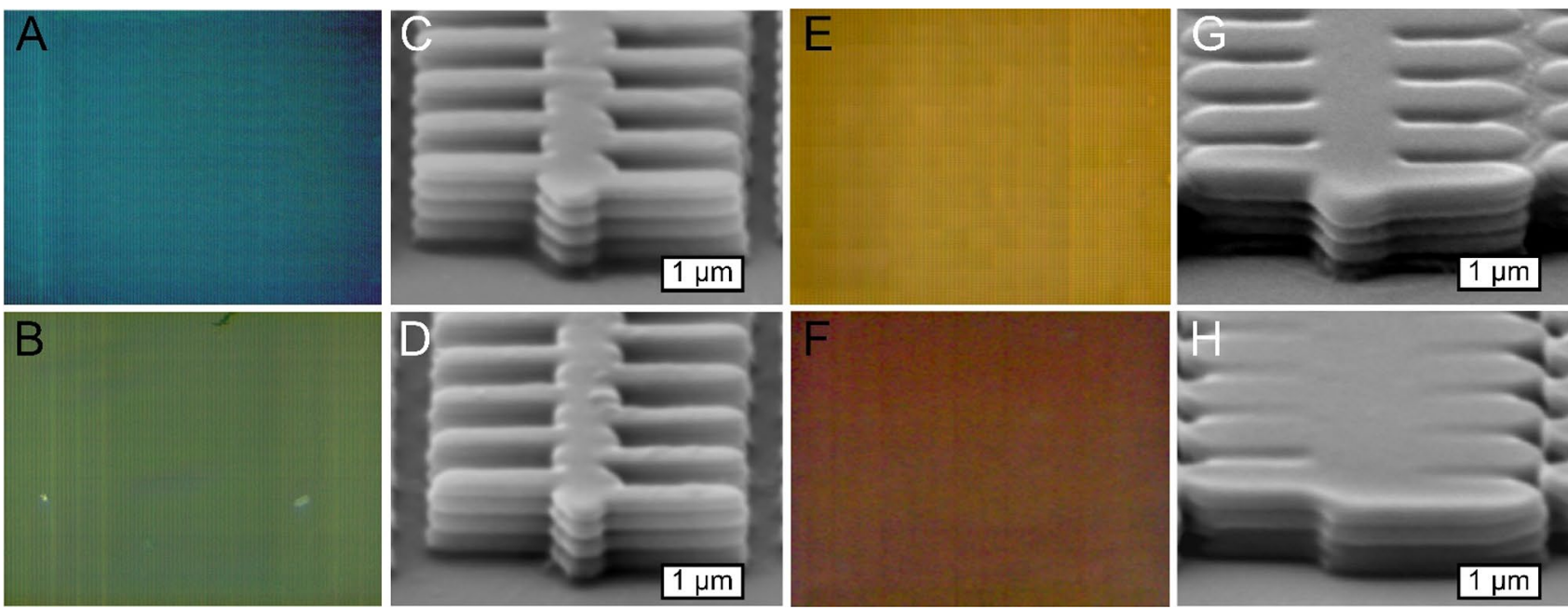

Fig. 2. Artificial structural colors resulted from biomimetic. a-h Illustration of blue, green, yellow, and brown structural color with corresponding SEM images of the multilayer systems produced. The

Table 1. Fabrication parameters for the production of individual structural color and the resulting layer dimensions of Femtobond 4B and air cavities

\begin{tabular}{llllll}
\hline Color & $P_{\mathrm{a}}[\mathrm{mW}]$ & $d_{1}[\mathrm{~nm}]$ & dis. amt. [\%] & $d_{2}[\mathrm{~nm}]$ & dis. amt. [\%] \\
\hline Blue & 10 & 85 & 15 & 110 & 25 \\
Green & 12 & 100 & 10 & 100 & 18 \\
Yellow & 16 & 140 & 0 & 20 & 0 \\
Brown & 19 & 170 & 0 & 5 & 0 \\
\hline
\end{tabular}

$P_{\mathrm{a}}, d_{1}, d_{2}$ refer to the average laser power used, the average thickness of a single polymer layer, and the average size of a single air cavity. Furthermore, the percentage amount of the variation of those dimensions referred to as disorder amount (dis. amt.) was examined with a SEM

values $d_{1}$ and $d_{2}$ were examined using ten polymer lines from five individual microstructures with the SEM (EVO MA 15, Zeiss, Jena, Germany). The disorder amount (dis. amt.) refers to the standard deviation.

The multilayer systems of the structures, which reflected the light in blue and green, were built from four layers of Femtobond 4B and four layers of air. In contrast, the multilayer systems corresponding to the yellow color were increased while the multilayer systems corresponding to the brown color were decreased by one layer of Femtobond 4B and air. The varying numbers of the multilayers can be attributed to the fluctuation of the photopolymer layer's thickness due to the limitation of the spin coater. Nevertheless, the color was not influenced by this difference, since the quantity of layers in a conventional multilayer system only influences the amount of reflection [16].

The different composition of the multilayer systems was due to the different average laser power values used for

multilayer systems differ due to the different average laser power used. The average laser power values and the examined size of single polymer layers and air cavities are provided in Table 1

structuring. The subfeatures in the cross-section of the line structures reflecting the color in blue were, consequently, the most obvious, as the lowest average laser power was used for their production (see Fig. 2c). Furthermore, disorder characteristics could be identified within these structures. Several polymer lines were not aligned entirely perpendicular to the cover glass surface but exhibited a tilt of up to $10^{\circ}$ (see Supplementary file). Additionally, similar to the lamellas of Morpho, the individual layer thicknesses of Femtobond 4B varied, and, therefore, the thickness of the air cavities also differed (see Supplementary file and Table 1 for values). These disorder characteristics were mainly caused by the low degree of polymerization [41] and the fluctuation of the average power of an ultrashort pulse laser [37, 42-44], since 2PP was performed near the polymerization threshold. In addition, impurities in the photosensitive material can also be attributed to the formation of the disorder characteristics.

Furthermore, the polymer lines showed slight height differences to each other (see Supplementary file), which can be explained by the low shrinkage properties of Femtobond 4B during the development process. These disorder characteristics can also be identified for the structures of the green-colored surface but to a lower degree due to the higher average laser power used for their production. Consequently, a further increase in the average laser power led to the successive reduction of the disorder characteristics. Therefore, the multilayer systems of the yellow and brown colored areas did not possess these properties, or they were so small that their dimensions could not be measured with the SEM. The impact of the disorder characteristics on the optical properties of the structural colors is analyzed in the following section. 


\subsection{Optical properties of artificial structural colors}

The optical properties of the structural colors presented in Fig. 2 were measured by angle-resolved spectroscopy using a self-built spectrometer. The set-up is shown in Zyla et al. [29]. Our colored surface was illuminated by a white light source (DH-2000-BAL, Ocean Optics Inc., Dunedin, Florida, United States) using a glass fiber (diameter $=200 \mu \mathrm{m})$ at an angle of $45^{\circ}$ referred to the surface normal of the structured area. The white light was focused by a condenser lens mounted on the glass fiber exit to a minimum diameter of $1 \mathrm{~mm}$ determined on the sample surface. The reflected radiation was captured by an opposite glass fiber of the same diameter and finally analyzed by a spectrometer (Ocean Optics Inc., Dunedin, FL, USA). The detector position or the position of the glass fiber, which detects the reflected light, could be varied in the angular range between $30^{\circ}$ and $60^{\circ}$, referring to the surface normal of the sample. The spectra were recorded with the software Spectra Suite ${ }^{\circledR}$ (Ocean Optics Inc, Dunedin, FL, USA). Further spectra processing included the dark noise subtraction, normalization of the spectra on the illumination source spectrum, smoothing with Savitzky-Golay [45], and normalization on the area of the spectra using MATLAB ${ }^{\circledR}$.

Furthermore, analytical calculations were run with MATLAB ${ }^{\circledR}$ using the respective layer thicknesses of the multilayer systems (see Table 1) to support our statement that the color formation contributes to multilayer interference. The analytical calculations were based on the Fresnel equations, which describe the proportional reflection and transmission of an electromagnetic plane wave reaching an interface between two loss-free dielectric media. The transfer-matrix method enables the calculation of this process to any number of layers $N$ with $N+1$ interfaces [46, 47]. Within the $N$ layers, this resulted in a formation of incoming (+) and outgoing ( - ) electromagnetic plane waves. Therefore, the amplitude values of the electric field can be determined at any interface $i$ between layer $i-1$ and layer $i$ within a multilayer system as described by Eq. 1 [47]. The amplitudes of the respective plane waves below the interface are marked with a line in the exponent. The values $\rho_{(i-1, i)}$ and $\tau_{(i-1, i)}$ refer to the reflection and transmission coefficient of the respective layers and can be calculated from the Fresnel equations $[46,47]$. Furthermore, the electromagnetic plane wave and the interface were infinitely extended so that diffraction effects were neglected:

$\left(\begin{array}{l}E_{0_{i-1}^{+}}^{+} \\ E_{0_{i-1}^{-1}}^{i^{\prime}}\end{array}\right)=\frac{1}{\tau_{i-1, i}}\left[\begin{array}{cc}1 & \rho_{\tau_{i-1, i}} \\ \rho_{\tau_{i-1, i}} & 1\end{array}\right]\left(\begin{array}{c}E_{0_{i}^{\prime}}^{\prime+} \\ E_{0_{i}}^{\prime-}\end{array}\right)=\boldsymbol{D}_{i-1, i}\left(\begin{array}{c}E_{0_{i}^{\prime}}^{\prime} \\ E_{0_{i}^{\prime}}^{\prime-}\end{array}\right)$.
The propagation of a plane wave through the layers $i-1$ can be described by Eq. 2 [47]:

$$
\left(\begin{array}{c}
E_{0_{i-1}^{\prime}}^{\prime+} \\
E_{0_{i-1}}^{\prime-1}
\end{array}\right)=\left[\begin{array}{cc}
e^{\left(-i \phi_{i-1}\right)} & 0 \\
0 & e^{\left(i \phi_{i-1}\right)}
\end{array}\right]\left(\begin{array}{l}
E_{0_{i-1}^{+}}^{+} \\
E_{0_{i-1}}^{-}
\end{array}\right)=\boldsymbol{P}_{i-1, i}\left(\begin{array}{l}
E_{0_{i-1}^{+}}^{+} \\
E_{0_{i-1}^{-1}}^{b^{-}}
\end{array}\right) .
$$

The complete description of the multilayer reflection is given by the transfer matrix, $\boldsymbol{M}=\boldsymbol{D}_{0} \prod_{i=1}^{N} \boldsymbol{P}_{i} \boldsymbol{D}_{i, i+1}$, which is calculated from the product of the individual transition and propagation matrices for the total number of layers $(i=1,2, \ldots, N)$ with $N+1$ interfaces [47]. Here, a multilayer system of the size $N+2$, assumes $N$ intermediate layers and two half-spaces for the description of the media outside the multilayer system, which, in our case, is air $(n=1)$. Therefore, the vector of the resulting amplitude values can be calculated by Eq. 3:

$\left(\begin{array}{c}E_{0}^{+} \\ E_{0}^{-}\end{array}\right)=M\left(\begin{array}{c}E_{0_{N+1}^{\prime+}}^{\prime+} \\ E_{0_{N+1}^{\prime}}^{\prime-}\end{array}\right)=\left[\begin{array}{ll}M_{11} & M_{12} \\ M_{21} & M_{22}\end{array}\right]\left(\begin{array}{c}E_{0_{N+1}^{\prime+}}^{\prime+} \\ E_{0_{N+1}^{\prime}}^{\prime-}\end{array}\right)$.

Finally, the reflectance, $R$, of a thin-film or multilayer system using unpolarized light is defined by Eq. 4. For this purpose, the mean value for the individual degrees of reflection was calculated from the perpendicular and parallel polarization direction of the electric field, taking into account the respective reflection and transmission coefficients from established Fresnel equations. The roughness of the surface was neglected:

$R=\frac{1}{2}\left|\frac{E_{0}^{-}}{E_{0}^{+}}\right|_{\perp}^{2}+\frac{1}{2}\left|\frac{E_{0}^{-}}{E_{0}^{+}}\right|_{\|}^{2}=\frac{1}{2}\left|\frac{M_{21}}{M_{11}}\right|_{\perp}^{2}+\frac{1}{2}\left|\frac{M_{21}}{M_{11}}\right|_{\|}^{2}$.

The results of the spectral measurements for each colored surface are shown in Fig. 3a, b. Additionally, the graphs are supplemented by the results from the analytical model highlighted in red. For the calculations, an angle of incidence of the plane waves on the multilayer systems of $45^{\circ}$ was defined. The calculated spectral response refers to the corresponding angle of reflection. To describe the multilayer systems, the estimated values from Table 1 were applied, including the respective disorder characteristics. A total number of $N=8$ alternating layers of Femtobond 4B $(n=1.51)$ and air $(n=1)$ was assumed. This number was increased to $N=10$, and reduced to $N=6$, Only for the calculation of the spectrum of the yellow and the brown color, respectively (see Fig. 2g, h).

Analytical results were compared with the spectral measurements where the angle of the incident was equal to the angle of reflection $\left(45^{\circ}\right)$. In this context, Fig. 3a illustrates that the color formation of blue and green was attributed mostly to multilayer interference. Notably, the analytical result for the green color matched the spectral 

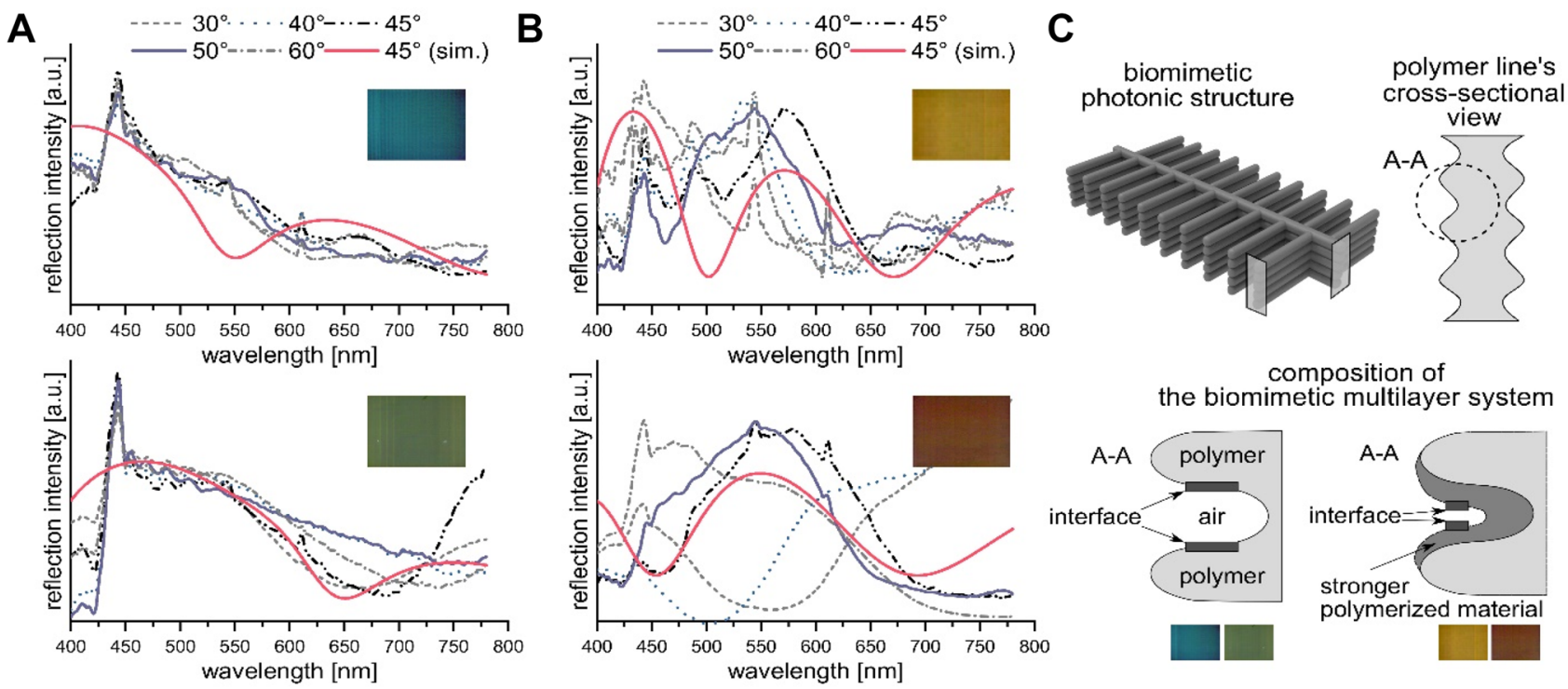

Fig. 3. Optical properties of artificial structural colors. a Graphs show angle-insensitive optical properties for the blue and green colored surfaces. b The optical properties of the yellow and brown colors differ in comparison to those shown in a. Here, the optical properties show characteristics of the conventional thin film- and multilayer interference. c Schematic illustration of interface sizereduction inside the multilayer systems due to higher average laser power used for structuring measurement. In contrast, the blue color's analytical result varied slightly from the measured spectrum since the model does not take into account all imperfections, e.g., the surface roughness of the generated structure. Moreover, every incident plane wave of different wavelength had the same amount of intensity in our analytical calculation, which did not correspond to reality. For example, conventional white light sources emit violet light only with low intensity. Therefore, the reflection intensity is decreased in the measured spectra in this wavelength range. Nevertheless, the dimensions of the multilayer system listed in Table 1, including its disorder characteristics are acceptable since the analytical model also matches the spectra for yellow and brown (see Fig. 3b). However, the optical properties differ between Fig. 3a, b considering the other detection angles of the spectral measurements. While the blue and green color have angle-insensitive optical properties in a range of $30^{\circ}$, this is not the case for yellow and brown. Only an explicit feature at approximately $440 \mathrm{~nm}$ is common for all of the graphs. This feature can be explained by Rayleigh scattering on the nanostructures in the cross-section of the polymer lines [29, 30, 48]. The angle-insensitivity of blue and green can be attributed to the disorder characteristics mentioned in Sec. 2.2. Specifically, the inhomogeneity inside the multilayer systems induces incoherent scattering as described by Kinoshita et al. [5].

The yellow and brown colors were more angle dependent because the disorder characteristics inside their surface structures were missing. Higher laser power led to larger polymerized structure size, thus the size of the air cavities was reduced. Simultaneously, the size of the interfaces was also reduced, as illustrated in Fig. 3c. Therefore, the interfaces of these multilayer systems were too small to produce the incoherent scattering [5]. Additionally, the higher average laser power provided the polymer lines with higher mechanical stability due to an increment of the structures' polymerization degree [41]. As a result, the line structures did not tilt on the cover glass.

\subsection{Application for artificial structural colors with angle-insensitive optical properties and molding of biomimetic 2PP structures}

Structural colors have a high potential for used in anticounterfeiting. The production of anticounterfeiting features usually requires sophisticated production methods and the use of various materials [49-51]. Consequently, the application of 2PP would have significant advantages, as described in the introduction.

For the generation of an anti-counterfeiting feature with $2 \mathrm{PP}$, the previous findings of our research were applied such that our structural colors only resulted from the interference system in the lines' cross-section [30] Therefore, various structure geometries with different symmetries (see Supplementary file) can be used to individually adjust the angleinsensitive optical properties in different tilting directions. For this purpose, structures referred to as arc structures were 
applied (see Fig. 4a) and orientated as shown in Fig. 4b. The process parameters listed in Table 1 were used to separately create a blue and green structural color (see Fig. 4c). Thus, Fig. $4 \mathrm{c}$ is a composite image that is only intended to illustrate how simply different color hues can be produced with 2PP. Here, all multilayer systems of the arc structures participated in the color formation process, regardless of their orientation, when the sample is not tilted under the light microscope. However, if the structured area was tilted around its vertical or horizontal axis $\left(40^{\circ}\right)$, the outer contour or the butterfly shape of the image appeared in the respective color (see Fig. 4d).

Furthermore, a second optical effect occurred when the structured area was analyzed in planar orientation using the same light microscope but in transmission (see Fig. 4e for the surface appearing in green). The results show that the structured surface has optical properties similar to an optical grating. Therefore, the perceived coloration in the transmission is based on diffraction since here, the gaps between the lines of the arc structure can be considered as individual slits. The intensity of the green color was reduced within the spectrum due to the optical properties in reflection. However, an interesting phenomenon is that the diffraction pattern varied depending on the illumination position. If the illumination was applied below or above the structured surface, the butterfly shape became apparent. In contrast, positioning the light source to the left or right of the structured surface caused a spectral coloration of the image's outer contour. This phenomenon can again be attributed to the different orientation of the arc structures in the overall structured area due to the partial symmetry of the arc structure, as specific slits only contribute to the color formation process depending on the illumination position.

Based on all of the presented results shown in Fig. 4, it can be concluded that biomimetic structures produced by 2PP are highly suitable for producing anticounterfeiting features due to the two separate effects in reflection and transmission. Furthermore, it is not only the simple production method, compared to multi-level manufacturing processes [49-51], which proves to be an advantage for the use of $2 \mathrm{PP}$. In this case, arbitrary images of anticounterfeiting attributes can also be easily generated without specific exposure masks. Specific structural geometries can adjust the optical properties of such features' different structural symmetry. Thus, a broad diversity of anticounterfeiting features produced by $2 \mathrm{PP}$ is conceivable.

However, the disadvantage of $2 \mathrm{PP}$ is the long production time required to fabricate photonic surface structures on a large scale. The structured area illustrated in Fig. 4, for example, measures $62.500 \mu \mathrm{m}^{2}$ and was produced in $3 \mathrm{~h}$. Consequently, 2PP processes do not represent an industrial standard. A potential method for scaling up our structured
A
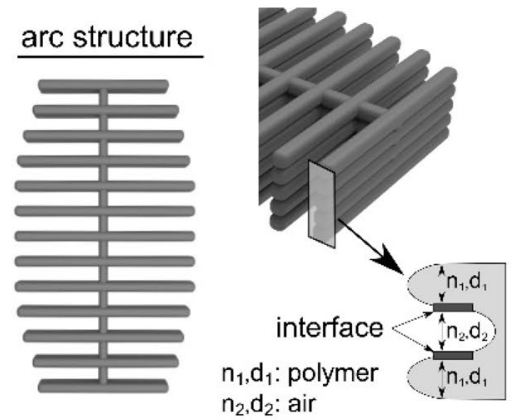

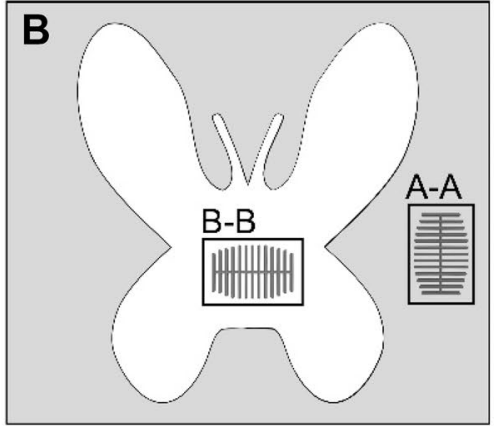
horizontal sample axis vertical sample axis
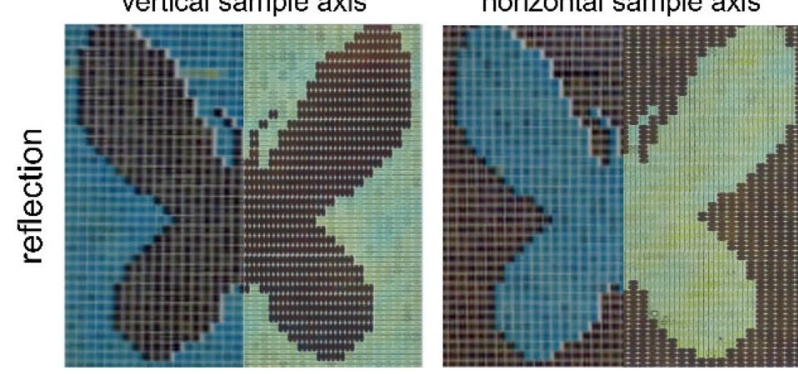

Fig. 4. Structural colors with angle-insensitive optical properties for anticounterfeiting. a Illustration of the arc structure with a modified cross-section of individual polymer lines, as presented in Fig. 1b, c. b Principal scheme for the fabrication of an anticounterfeit feature using a specific orientation of the arc structure in the overall structhe illumination position
E

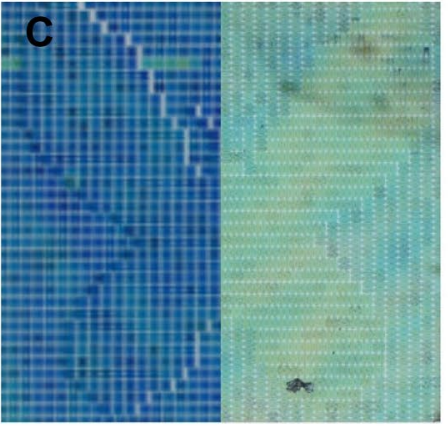

light source position on the right of the structuring
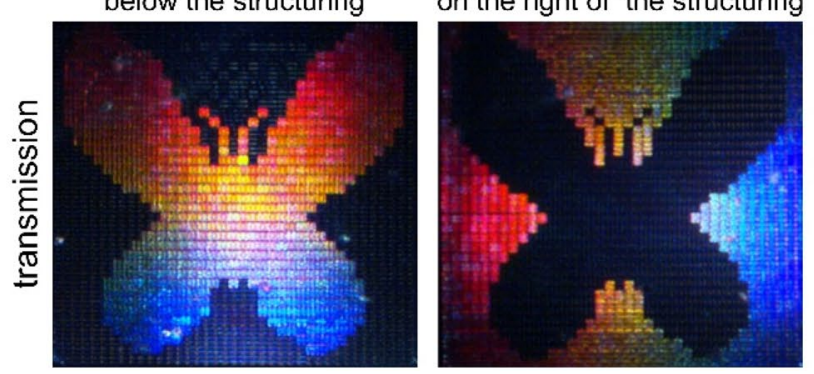

tured area. c Coloration of planar aligned biomimetic surface structures. For the generation of these colors, the parameters from Table 1 were used. d, e Optical properties of biomimetic surface structures in reflection and transmission depend on the samples' tilt direction or 
surfaces is to mold them. Molding is especially common in biology to reproduce organic surface structures with high resolution [52]. The 2PPstructured surfaces serve as a master, which can be used to generate an impression in several minutes. This procedure can be repeated, in principle, multiple times because, 2PP structures are usually highly robust [53]. Therefore, multiple separated replicas can be fabricated from the impressions in one process step, and then each replica can be fixed on a base substrate to create larger structured surfaces. However, each replica must be evenly cut to size before it is affixed. Furthermore, the surface of the ground material has to be uniformly smooth. While this approach merely describes the main idea of how the enlargement of 2PP-structured surfaces is feasible in principle, it has to initially prove that the small substructures in the cross-section of the polymer lines can be replicated. Therefore, we performed the molding process, as illustrated in Fig. 5a on biomimetic structures that reflect the light in the blue (see Fig. 5b inset, $A=62.500 \mu \mathrm{m}^{2}$ ). A negative of the biomimetic surface structuring was produced using a two-component dental wax (President, The Original Light Body, Coltene, Switzerland), which contains mainly polyvinylsiloxane. An equal ratio of both components was manually applied to the 2PP structures using a standard cartridge gun (President, The Original Light Body, Coltene, Switzerland). To later obtain a flat surface of the replica/ positive, the dental wax was manually pressed with a cover glass after its application. Curing of the dental wax was performed in the air for approximately $5 \mathrm{~min}$. Afterwards, the negative was carefully removed. Dental wax was also applied around the negative produced so that it could be filled with a polymer in the next step. For the replica production, the epoxy resin Spurr [54] was used, which has a refractive index of approximately $n=1.525$ and a slightly yellowish color after polymerization. Spurr was cured for 6-8 $\mathrm{h}$ in an oven at a temperature of $70{ }^{\circ} \mathrm{C}$.

The optical result for the replica is shown in Fig. 5b. Since individual line structures of the replica also exhibited multilayer systems in their cross-section (see Fig. 5c), the color formation can again be attributed to multilayer interference. Nevertheless, there was a shift in the color hue from blue to green, which may have been caused by manually applied pressure with a cover glass during the negative preparation. Therefore, the interference system in the crosssection of the individual lines in the negative was modified. An additional result in the supplementary file demonstrated that this hypothesis is probable. Here, it could be proved that the color hue's shift is not due to Spurr's optical properties because the same color from the original can be reproduced. However, in this case, some replica structures were damaged during the manually performed removal from the negative. Therefore, the intensity of the color in these results is rather low.

Furthermore, the optical properties of the replica were analyzed shown in Fig. 5b, again using angle-resolved spectroscopy. The spectra measured are illustrated in Fig. 5d which shows that the replica's structural color is also angleinsensitive in the same range as the original. Therefore, it can be concluded that the multilayer systems of the replica have disorder characteristics comparable to those of blue Morpho butterflies [14, 16, 33-35].
A (1) Molding of biomimetic 2PP structures

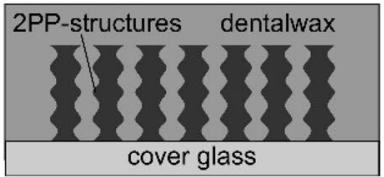

(2) Biomimetic negative

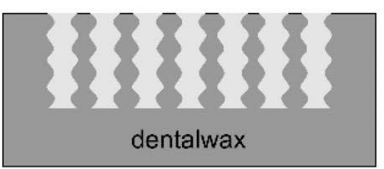

(3) Molding of biomimetic

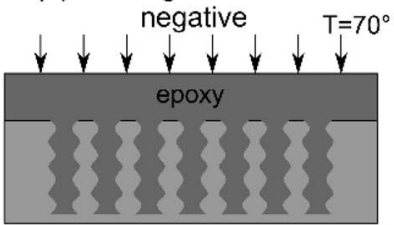

(4) Biomimetic replica

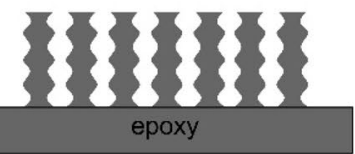

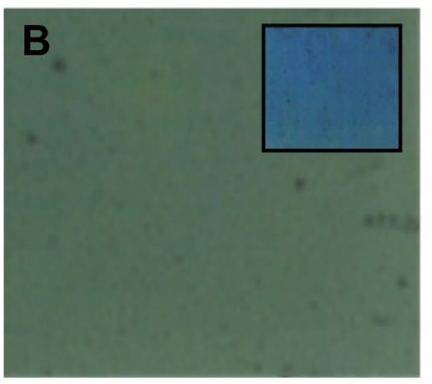
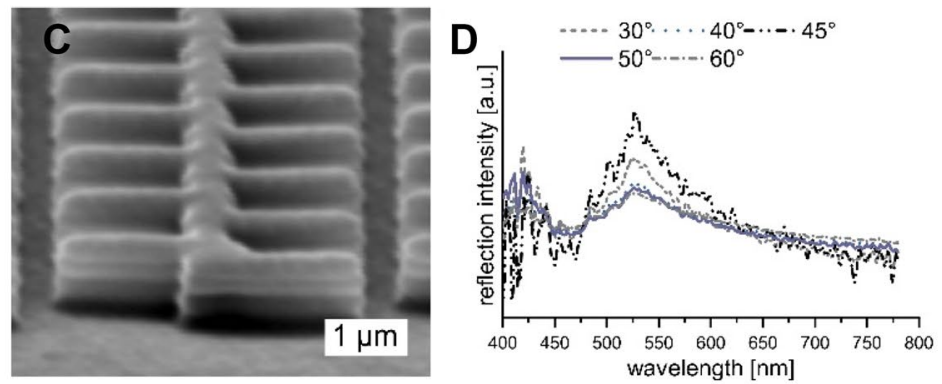

Fig. 5. Molding of biomimetic $2 \mathrm{PP}$ structures. a Schematic illustration of the procedure of molding biomimetic 2PP structures. b Illustration of the replica's structural color. The color of the original structured area is shown in the inset. c Illustration of the cross-section of

replica line structures generated, which are similar to the original. d The structural color of the replica also exhibits angle-insensitive optical properties examined by angle-resolved spectroscopy 


\section{Discussion and conclusion}

The results presented further extend our research work in manufacturing structural colors inspired by nature with angle-insensitive optical properties mimicking the surface structures of the blue butterflies from the genus Morpho with 2PP [29, 30]. In this work, we used the interference-assisted polymerization method $[38,39]$ to create different biomimetic multilayer systems which generated different colors. The multilayer systems were modified by varying only the average laser power used for structuring. In this case, we showed that the multilayer features could be adjusted in the range of approximately $20 \mathrm{~nm}$, whereas the conventional $2 \mathrm{PP}$ process is subject to a lower resolution [37]. The 2PP approach presented is accurate and reproducible (see Supplementary file) so that different colors can also be fabricated even on a single glass substrate.

However, while measuring the optical properties of different structural colors, it became apparent that only the 2PP structures of the blue and green colored surfaces (see Fig. 3a) exhibited angle-insensitive optical properties in a range of $30^{\circ}-40^{\circ}$ due to their Morpho-like disorder characteristics $[14,16,33-35]$. The existence of disorder inside the multilayer system was further proven by analytical calculation. These disorder characteristics can be attributed to the degree of polymerization [41], the fluctuations in the average laser power of ultrashort pulse lasers near the polymerization threshold [37, 42-44], the shrinkage of Femtobond 4B and impurities in the photosensitive material. This circumstance only randomly influence the $2 \mathrm{PP}$ process, but the production of the disorder characteristics is reproducible, as shown in Ref. [29] and Ref. [30]. Therefore, the current state of the literature again confirmed again that the disorder in the photonic system of the blue Morpho butterflies is essential to reflect the light angle-insensitivity [14, 16, 33-35].

Additionally, the findings gained from the previous results and those presented here serve to open up application possibilities in the area of anticounterfeiting. For this purpose, the fact that our angle-intensive structural colors do solely depend on the multilayer system generated [30] (see Supplementary file). Therefore, a diverse orientation of partly symmetrical biomimetic structures results in different optical appearances in the reflection, depending on the sample tilt direction. Furthermore, coloration occurs in transmission due to diffraction, as in the cover scales of the blue Morpho butterflies [16]. The optical appearance was additionally related to the position of the illumination, which can again be attributed to the different orientation of biomimetic photonic structures.

Finally, a low-cost and straightforward molding technique was used to generate replicas of the original $2 \mathrm{PP}$ structures. This approach is mainly established in biology to create surface structures replicas [52]. In this context, we could show that even the substructures in the crosssection of individual polymer lines could be molded. Furthermore, the replicas exhibited the same disorder characteristics, which ensures that the replicas' optical properties were also angle-insensitive. Therefore, the molding technique offers a great opportunity for scaling up 2PP-generated surface structures.

In conclusion, 2PP is not only a suitable research tool. Indeed, 2PP also has excellent potential for the generation of blueprints as a basis for the development of potential realworld applications [55-58].

Electronic supplementary material The online version of this article (https://doi.org/10.1007/s00339-020-03931-6) contains supplementary material, which is available to authorized users.

Acknowledgements This publication was supported by the Ruhr University Research School PLUS, funded by Germany's Excellence Initiative [DFG GSC 98/3].

Funding Open Access funding provided by Projekt DEAL.

\section{Compliance with ethical standards}

Conflict of interest The authors declare that there is no conflict of interests regarding the publication of this manuscript.

Informed consent All of the coworkers have agreed to participate.

Open Access This article is licensed under a Creative Commons Attribution 4.0 International License, which permits use, sharing, adaptation, distribution and reproduction in any medium or format, as long as you give appropriate credit to the original author(s) and the source, provide a link to the Creative Commons licence, and indicate if changes were made. The images or other third party material in this article are included in the article's Creative Commons licence, unless indicated otherwise in a credit line to the material. If material is not included in the article's Creative Commons licence and your intended use is not permitted by statutory regulation or exceeds the permitted use, you will need to obtain permission directly from the copyright holder. To view a copy of this licence, visit http://creativecommons.org/licenses/by/4.0/.

\section{References}

1. W. Wickler, Mimicry and the evolution of animal communication. Nature 208, 519-521 (1965)

2. L.M. Arenas, J. Troscianko, M. Stevens, Color contrast and stability as key elements for effective warning signals. Front. Ecol. Evol. 2, 1544 (2014)

3. T.D. Schultz, O.M. Fincke, Structural colours create a flashing cue for sexual recognition and male quality in a neotropical giant damselfly. Funct. Ecol. 23, 724-732 (2009)

4. S. Kinoshita (ed.), Pattern Formations and Oscillatory Phenomena: 6. Structural Color in Nature: Basic Observations and Analysis (Elsevier, Amsterdam, 2013) 
5. S. Kinoshita, Structural colors in the realm of nature (WorldScientific, Singapore, 2008)

6. P. Vukusic, J.R. Sambles, Photonic structures in biology. Nature 424, 852-855 (2003)

7. K. Yu, T. Fan, S. Lou, D. Zhang, Biomimetic optical materials: integration of nature's design for manipulation of light. Prog. Mater. Sci. 58, 825-873 (2013)

8. J. Sun, B. Bhushan, J. Tong, Structural coloration in nature. RSC Adv. 3, 14862 (2013)

9. V.E. Johansen, O.D. Onelli, L.M. Steiner, S. Vignolini, Photonics in nature: from order to disorder, in Functional Surfaces in Biology III. Biologically-Inspired Systems, vol. 10, ed. by S. Gorb, E. Gorb (Springer, Cham, 2017)

10. P. Vukusic, B. Hallam, J. Noyes, Brilliant whiteness in ultrathin beetle scales. Science 315, 348-348 (2007)

11. M. Spinner, A. Kovalev, S.N. Gorb, G. Westhoff, Snake vel-vet black: hierarchical micro- and nanostructure enhances dark colouration in bitis rhinoceros. Sci. Rep. 3, 1846 (2013)

12. M. Spinner, S.N. Gorb, A. Balmert, H. Bleckmann, G. Westhoff, Non-contaminating camouflage: multifunctional skin microornamentation in the west african gaboon viper (bitisrhinoceros). PLoS ONE 9, e91087 (2014)

13. V. Sharma, M. Crne, J.O. Park, M. Srinivasarao, Structural origin of circularly polarized iridescence in jeweled beetles. Science $\mathbf{3 2 5}$, 449-451 (2009)

14. P. Vukusic, J.R. Sambles, C.R. Lawrence, R.J. Wootton, Quantified interference and diffraction in single Morpho butterfly scales. Proc. Roy. Soc. Lond. B Biol. Sci. 266, 1403-1411 (1999)

15. S. Kinoshita, S. Yoshioka, K. Kawagoe, Mechanisms of structural colour in the Morpho butterfly: cooperation of regularity and irregularity in an iridescent scale. Proc. Roy. Soc. Lond. B Biol. Sci. 269, 1417-1421 (2002)

16. S. Kinoshita, S. Yoshioka, J. Miyazaki, Physics of structural colors. Rep. Prog. Phys. 71, 076401 (2008)

17. S. Yoshioka, S. Kinoshita, Wavelength-selective and anisotropic light-diffusing scale on the wing of the Morpho butterfly. Proc. Roy. Soc. Lond. B Biol. Sci. 271, 581-587 (2004)

18. H. Ghiradella, Light and color on the wing: structural colors in butterflies and moths. Appl. Optics 30, 3492-3500 (1991)

19. G. Shang, M. Eich, A. Petrov, Photonic glassbased structural color. APL Photonics 5, 060901 (2020)

20. S. Maragkaki, C.A. Skaradzinski, R. Nett, E.L. Gurevich, Influence of defects on structural colours generated by laserinduced ripples. Sci. Rep. (2017). https://doi.org/10.1038/ s41598-019-56638-x

21. R.H. Siddique, R. Hünig, A. Faisal, U. Lemmer, H. Hölscher, Fabrication of hierarchical photonic nanostructures inspired by Morpho butterflies utilizing laser interference lithography. Opt. Mater. Express 5, 996 (2015)

22. R.H. Siddique, S. Diewald, J. Leuthold, H. Hölscher, Theoretical and experimental analysis of the structural pattern responsible for the iridescence of Morpho butterflies. Opt. Express 21, 1435114361 (2013)

23. R.A. Potyrailo, R.K. Bonam, J.G. Hartley, T.A. Starkey, P. Vukusic, M. Vasudev, T. Bunning, R.R. Naik, Z. Tang, M.A. Palacios, M. Larsen, L.A. Le Tarte, J.C. Grande, S. Zhong, T. Deng, Towards outperforming conventional sensor arrays with fabricated individual photonic vapour sensors inspired by Morpho butterflies. Nat. Commun. 6, 7959 (2015)

24. S. Zhang, Y. Chen, Nanofabrication and coloration study of artificial Morpho butterfly wings with aligned lamellae layers. Sci. Rep. 5, 16637 (2015)

25. K. Chung, S. Yu, C.-J. Heo, J.W. Shim, S.-M. Yang, M.G. Han, H.-S. Lee, Y. Jin, S.Y. Lee, N. Park, J.H. Shin, Flexible angleindependent, structural color reflectors inspired by Morpho butterfly wings. Adv. Mater. 24, 2375-2379 (2012)
26. B. Song, V.E. Johansen, O. Sigmund, J.H. Shin, Reproducing the hierarchy of disorder for Morpho-inspired, broad-angle color reflection. Sci. Rep. 7, 46023 (2017)

27. B.-K. Hsiung, R.H. Siddique, L. Jiang, Y. Liu, Y. Lu, M.D. Shawkey, T.A. Blackledge, Tarantula-inspired noniridescent photonics with long-range order. Adv. Opt. Mater. 5, 1600599 (2017)

28. B.-K. Hsiung, R.H. Siddique, D.G. Stavenga, J.C. Otto, M.C. Allen, Y. Liu, Y.-F. Lu, D.D. Deheyn, M.D. Shawkey, T.A. Blackledge, Rainbow peacock spiders inspire miniature super-iridescent optics. Nat. Commun. 8, 2278 (2017)

29. G. Zyla, A. Kovalev, M. Grafen, E.L. Gurevich, C. Esen, A. Ostendorf, S. Gorb, Generation of bioinspired structural col-ors via two-photon polymerization. Sci. Rep. 7, 17622 (2017)

30. G. Zyla, A. Kovalev, S. Heisterkamp, C. Esen, E.L. Gurevich, S. Gorb, A. Ostendorf, Biomimetic structural colorationwith tunable degree of angle-independence generated by two-photon polymerization. Opt. Mater. Express 9, 2630 (2019)

31. Y. Liu, W. Xiong, D.W. Li, Y. Lu, X. Huang, H. Liu, L.S. Fan, L. Jiang, J.-F. Silvain, Y.F. Lu, Precise assembly and joining of silver nanowires in three dimensions for highly conductive composite structures. Int. J. Extrem. Manuf. 1, 025001 (2019)

32. K. Sugioka, Hybrid femtosecond laser three-dimensional microand nanoprocessing: a review. Int. J. Extrem. Manuf. 1, 012003 (2019)

33. K. Watanabe, T. Hoshino, K. Kanda, Y. Haruyama, S. Matsui, Brilliant blue observation from a Morphobutterfly-scale quasistructure. Jpn. J. Appl. Phys. 44, 48-50 (2005)

34. S. Kinoshita, S. Yoshioka, Y. Fuji, N. Okamoto, Photo-physics of structural color in the Morpho butterflies. Forma 17, 103-121 (2002)

35. Y. Ding, S. Xu, Z.L. Wang, Structural colors from Morpho peleides butterfly wing scales. J. Appl. Phys. 106, 074702 (2009)

36. H. Ghiradella, "Hairs, bristles and scales, in Microscopic anatomy of invertebrates, Vol. 11: insecta, ed. by F.W. Harrison, M. Locke (Wiley, New York, 1998)

37. J. Serbin, A. Egbert, A. Ostendorf, B.N. Chichkov, R. Hou-bertz, G. Domann, J. Schulz, C. Cronauer, L. Fröhlich, M. Popall, Femtosecond laser-induced two-photon polymerization of inorganicorganic hybrid materials for applications in photonics. Opt. Lett. 28, 301-303 (2003)

38. B. Mills, D. Kundys, M. Farsari, S. Mailis, R.W. Eason, Singlepulse multiphoton fabrication of high aspect ratio structures with sub-micron features using vortex beams. Appl. Phys. A 108, 651-655 (2012)

39. Q.-Q. Liu, Y.-Y. Zhao, M.-L. Zheng, X.-M. Duan, Tunable multilayer submicrostructures fabricated by interference assisted twophoton polymerization. Appl. Phys. Lett. 111, 223102 (2017)

40. M. Born, E. Wolf, Principles of optics: electromagnetic theory of propagation, interference and diffraction of light, 7th edn. (Cambridge University Press, Cambridge, 2000)

41. Y. Liu, J.H. Campbell, O. Stein, L. Jiang, J. Hund, Y. Lu, Deformation behavior of foam laser targets fabricated by two-photon polymerization. Nanomater. Basel 8, 498 (2018)

42. J. Fischer, M. Wegener, Three-dimensional optical laser lithography beyond the diffraction limit. Laser Photonics Rev. 7, 22-44 (2013)

43. K. Takada, H.-B. Sun, S. Kawata, Improved spatial resolution and surface roughness in photopolymerization-based laser nanowriting. Appl. Phys. Lett. 86, 071122 (2005)

44. X. Zhou, Y. Hou, J. Lin, A review on the processing accuracy of two-photon polymerization. AIP Adv. 5, 030701 (2015)

45. A. Savitzky, M.J.E. Golay, Smoothing and differentiation of data by simplified least squares procedures. Anal. Chem. 36, 16271639 (1964)

46. X. Rui, G. Wang, J. Zhang, Transfer Matrix Method for Multibody Systems: Theory and Applications (Wiley, Hoboken, 2018) 
47. C.C. Katsidis, D.I. Siapkas, General transfer-matrix method for optical multilayer systems with coherent, partially coherent, and incoherent interference. Appl. Optics 41, 3978-3987 (2002)

48. Rayleigh, XV. On the theory of optical images, with specialreference to the microscope. Philos. Mag. 42, 167-195 (1896)

49. G. Ruffato, R. Rossi, M. Massari, E. Mafakheri, P. Capaldo, F. Romanato, Design, fabrication and characterization of computer generated holograms for anticounterfeiting applications using oam beams as light decoders. Sci. Rep. 7, 18011 (2017)

50. R. Arppe, T.J. Sørensen, Physical unclonable functions generated through chemical methods for anti-counterfeiting. Nat. Rev. Chem. 1, 0031 (2017)

51. H. Nam, K. Song, D. Ha, T. Kim, Inkjet printing based mono-layered photonic crystal patterning for anti-counterfeiting structural colors. Sci. Rep. 6, 30885 (2016)

52. S.N. Gorb, Visualisation of native surfaces by two-step molding. Microsc. Today 15, 44-47 (2007)

53. J.F. Busche, G. Starke, S. Knickmeier, A. Dietzel, Controllable dry adhesion based on two-photon polymerization and replication molding for space debris removal. Micro Nano Eng. 7, 100052 (2020)

54. A.R. Spurr, A low-viscosity epoxy resin embedding medium forelectron microscopy. J. Ultrastruct. Res. 26, 31-43 (1969)
55. H. Xing, J. Li, Y. Shi, J. Guo, J. Wei, Thermally driven photonic actuator based on silica opal photonic crystal with liquid crystal elastomer. ACS Appl. Mater. Inter. 8, 9440-9445 (2016)

56. J. Mu, G. Wang, H. Yan, H. Li, X. Wang, E. Gao, C. Hou, A.T.C. Pham, L. Wu, Q. Zhang, Y. Li, Z. Xu, Y. Guo, E. Reichmanis, H. Wang, M. Zhu, Molecular-channel driven actuator with considerations for multiple configurations and color switching. Nat. Commun. 9, 590 (2018)

57. L. Gao, Y. Zhang, V. Malyarchuk, L. Jia, K.-I. Jang, R.C. Webb, H. Fu, Y. Shi, G. Zhou, L. Shi, D. Shah, X. Huang, B. Xu, C. Yu, Y. Huang, J.A. Rogers, Epidermal photonic devices for quantitative imaging of temperature and thermal transport characteristics of the skin. Nat. Commun. 5, 4938 (2014)

58. Y. Fu, C.A. Tippets, E.U. Donev, R. Lopez, Structural colors: from natural to artificial systems. WIREs Nanomed. Nanobiotechnol. 8, 758-775 (2016)

Publisher's Note Springer Nature remains neutral with regard to jurisdictional claims in published maps and institutional affiliations. 